\title{
Anatomy, Biomechanics and Complications of Arthroscopic Anterior Cruciate Ligament Reconstruction
}

\author{
Kamal Abdel Rahman AbdelHafez, Mohammed AbdelAziz Hassan, Wael Said Altaher Mohammed
}

\begin{abstract}
Department of Orthopedic Surgery, Faculty of Medicine, Al-Azhar University, Cairo, Egypt
Corresponding author: Wael Said Altaher Mohammed, Mobile: 00201149452945, email: dr.wael86@yahoo.com

Abstract

Background: With increasing road traffic accidents and athletic participation, traumatic lesions of the ligaments about the knee are becoming increasingly more common. Those injuries can be isolated or combined with other components of the knee. No procedure is ever completely free of risks. However, Anterior Cruciate Ligament (ACL) reconstruction is a safe procedure that is performed thousands of times each year. ACL reconstruction surgery uses a graft to replace the ligament. The most common grafts are autografts using part of body, such as patellar tendon or one of the hamstring tendons. Results of arthroscopic ACL reconstruction have been excellent; the success rate has been reported to be more than $95 \%$ in term of patient satisfaction. Aim of the work: was to discuss anatomy and biomechanics of anterior cruciate ligament and the complications of arthroscopic anterior cruciate ligament reconstruction. Conclusion: It could be concluded that the challenge is to perform the reconstructive procedure with high success and minimal complications and morbidity. To accomplish this, it requires a combination of a skilled surgeon, appropriate graft selection and careful attention to the details of rehabilitation.
\end{abstract}

Keywords: Arthroscopic, Anterior cruciate ligament Introduction:

Arthroscopy of the knee was first performed in 1918 when Kenji Takagi used a cystoscope to examine the intra-articular structures of the knee ${ }^{(\mathbf{1})}$. Burman, at the Hospital for Joint Diseases in New York, reported on his arthroscopic study of cadaveric joints, including the knee, in $1931^{(2)}$. He concluded that arthroscopy should be used for purposes of diagnosis rather than traditional open exploratory surgery. His report was met with little enthusiasm by his medical colleagues.

Arthroscopic surgery of the knee evolved slowly over the years. The first arthroscopic knee surgery was performed by Masaki Watanabe in 1955. The procedure involved a partial excision of pigmented villonodular synovitis. Watanabe performed the first arthroscopic meniscectomy ${ }^{(3)}$. Arthroscopic techniques were pioneered by Bradley ${ }^{(4)}$. Over the past 25 years, the arthroscope has revolutionized the diagnosis and surgical treatment of disorders of the knee. The future holds nothing but promise for further advancements in treatment of the knee, as well as other joints ${ }^{(4)}$.

ACL reconstruction has become a great challenge and many techniques has been evolved and created according to way of graft fixation such as metal and biodegradable interference screws, staples, buttons, press-fit. The success of the surgery depends on several factors including the timing of the surgery, graft choice, tunnel placement, graft tensioning, graft fixation methods, and postoperative rehabilitation protocol. Orthopedic surgeons use bone-patellar tendon-bone or hamstring tendon grafts most

frequently. Secure graft fixation is an important factor, especially in the early postoperative period ${ }^{(5)}$.

Complications associated with this surgery occur in $1.8 \%$ to $24 \%$ of the procedures, which include joint stiffness, patellar fracture, infection, hardware failure, graft failure, wound complications, deep vein thrombosis and periarticular fractures. A rare and devastating complication following ACL reconstruction and revision reconstruction is femoral fracture ${ }^{(5)}$.

Patella fracture after anterior cruciate ligament reconstruction of the knee can be a serious problem in the total rehabilitation of the patient, and that possibility should be considered during anterior cruciate ligament reconstruction. These complications can be prevented by avoiding taking too much bone graft, by using the most precise tools for cutting, while rehabilitation must be carefully planned ${ }^{(6)}$.

Aim of the work was to discuss anatomy and biomechanics of anterior cruciate ligament and discuss the complications of arthroscopic anterior cruciate ligament reconstruction.

Anatomy

\section{ANATOMY OF ACL}

The knee joint starts its formation from vascular mesenchyme between femur and tibia in the fourth week of gestation between the blastoma of femur and tibia. A distinct amount of ACL fibers appear in approximately the 8th week after gestation. At this time, the fibroblasts within the ligament are already aligned to the axis of strain of the $\operatorname{ACL}^{(7,8)}$.

By 9 weeks, the cruciate ligaments are composed of numerous immature fibroblasts having 


\section{Kamal AbdelHafez et al.}

scanty cytoplasm and fusiform nuclei. Over the next weeks, the major change in addition to growth, is the increase in vascularity. During this time the fusiform fibroblasts express high amounts of the angiogenic vascular endothelial growth factor ${ }^{(9)}$ (Fig. 1).

The expression of vascular endothelial growth factor is largely downregulated after birth, but

A

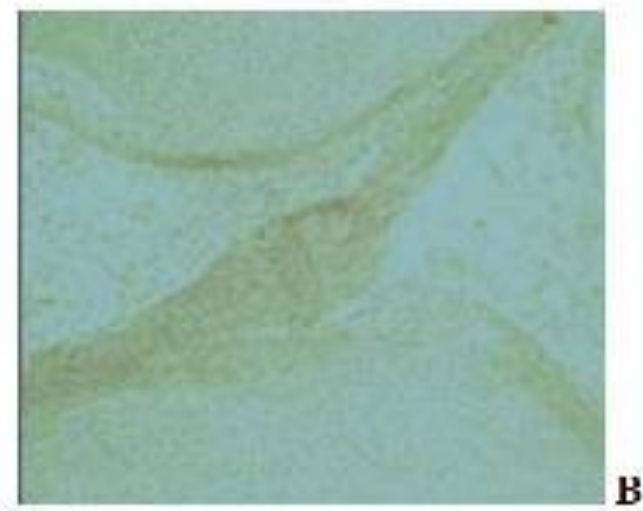

this factor is strongly re-expressed during the remodeling of autologous tendon grafts used for ACL reconstruction. After week 20, the remaining development consists of marked growth with little change in form. ${ }^{(10)}$ Two distinct bundles of the ACL are present at 16 weeks of gestation ${ }^{(\mathbf{1 1})}$ (Fig. 2).

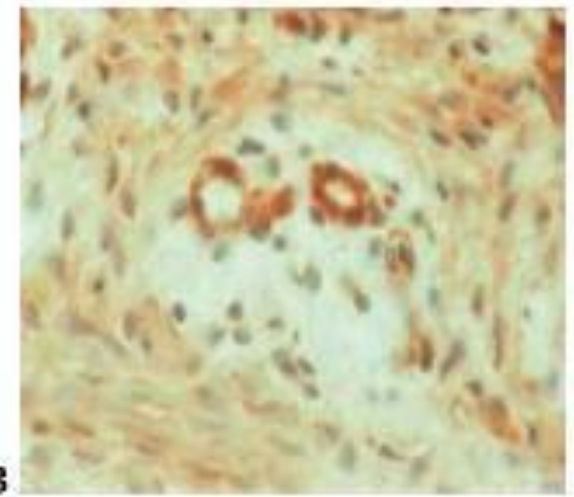

Fig. (1): (A) Fetal ACL,10th week after gestation. Note the early organization of the ACL between femur and tibia. (B) Vascular endothelial immunostaining, fetal ACL 22 weeks after gestation ${ }^{\text {(12) }}$.
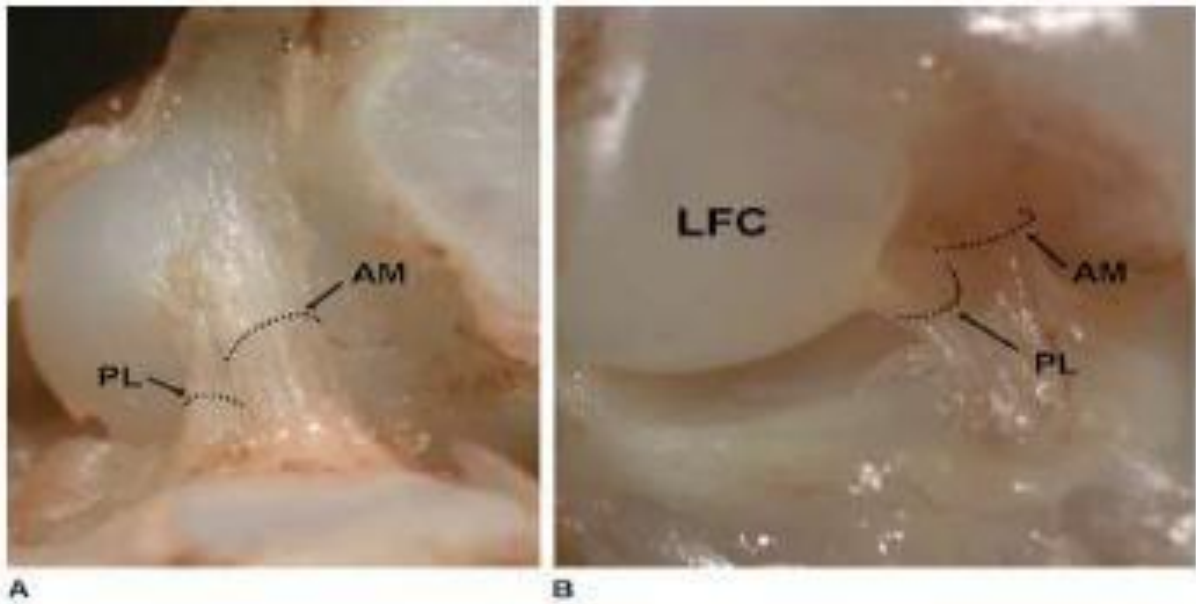

Fig. (2): 16-week fetus demonstrating two bundles of the anterior cruciate ligament with the knee in extension (A, sagittal view with medial femoral condyle removed) and flexion (B, frontal view). AM, Anteromedial; LFC, lateral femoral condyle; PL, posterolateral ${ }^{(\mathbf{1 3})}$

\section{Gross Anatomy:}

The ACL is a band-like structure of dense connective tissues. Its femoral attachment displays a shape comparable to a vertically disposed semicircle, the ACL must be regarded only as an intraarticular structure because it can be reconstructed only intraarticularly and cannot be exteriorized from the articular space ${ }^{\mathbf{( 1 2 )}}$.

The ligament originates at the medial side of the lateral femoral condyle and runs an oblique course through the intercondylar fossa distal-anteriormedial to the insertion at the medial tibial eminence. The axis of the long diameter of the ACL is tilted $26^{\circ}$ $\pm 6^{\circ}$ forward from the vertical. When the knee is flexed, as during surgery, the ligament seems to turn itself in a lateral spiral ${ }^{(\mathbf{1 3})}$.

This external rotation is approximately $90^{\circ}$ as the fibers approach the tibial surface. The twist of the fibers of the ACL is a result of the orientation of its bony attachments. The femoral attachment is oriented primarily in the longitudinal axis of the femur, whereas the tibial attachment is in the anteroposterior axis of the tibia ${ }^{(13)}$ (Fig. 3).

The ACL length ranges from 22 to $41 \mathrm{~mm}$ (mean, $32 \mathrm{~mm}$ ) and its width from 7 to $12 \mathrm{~mm}$, mean length of the AM bundle is $33 \mathrm{~mm}$ and is $18 \mathrm{~mm}$ for the PL bundle ${ }^{(\mathbf{1 4 , 1 5})}$.

The narrowest diameter of the ACL occurs in the midsubstance which is oval in shape with an area of $36 \mathrm{~mm}^{2}$ and $44 \mathrm{~mm}^{2}$ for females and males, respectively. The insertion areas of the ACL (tibial and femoral insertion), were found to be over 3.5 times larger than the ligament midsubstance. The broad femoral and tibial insertion area with respect to the narrow diameter of the midsubstance makes selection 
of tunnel site placement challenging because of the limited size of single-bundle grafts ${ }^{(14,15)}$ (Fig. 4).

The ACL tibial attachment fans out and forms a "foot" region which has been found to be both larger and stronger than the femoral. This allows the ACL to tuck under the roof of the intercondylar notch. In full extension the anterior fibers of the ACL turn around the anterior edge of the intercondylar notch. This bending is considered as a "physiological impingement" (16).
This specific anatomy causes concern for ACL reconstruction since common grafts do not posses such a "foot"-type region. If a straight graft is inserted in the anterior part of the tibial ACL insertion it tends to impinge with the notch in slight degrees of flexion. Notch impingement due to anterior tibial tunnel positions is a common cause for postoperative extensions block ${ }^{(\mathbf{1 7})}$.

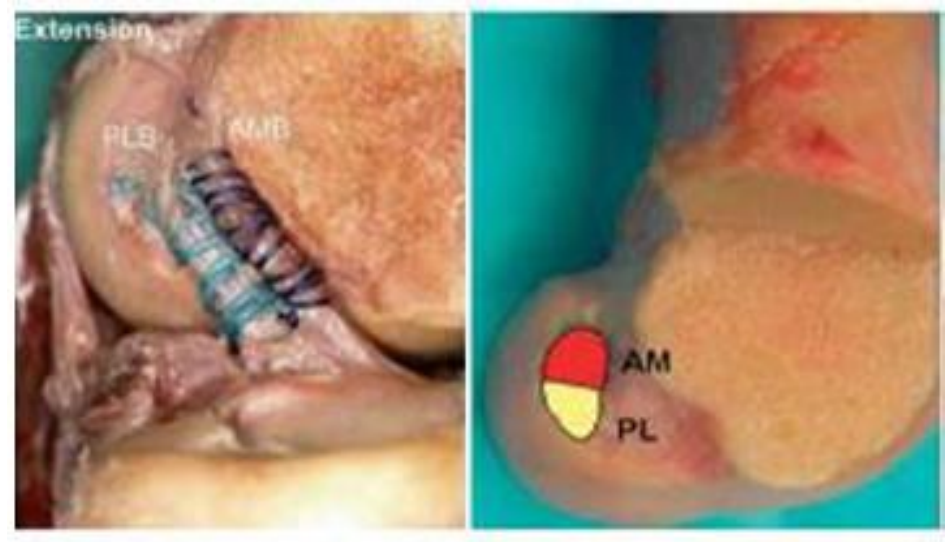

A

B

(A) With the knee in extension, the AM and PL bundles are parallel (left knee, medial femoral condyle removed) and the insertion sites are oriented vertically (left).

(B) With the knee in flexion, the AM and PL bundles are crossed (A, left knee, medial femoral condyle removed) and the insertion sites are oriented horizontally (right).

Fig. (3): Crossing pattern of anteromedial (AM) and posterolateral (PL) bundles ${ }^{\mathbf{1 5}}$.

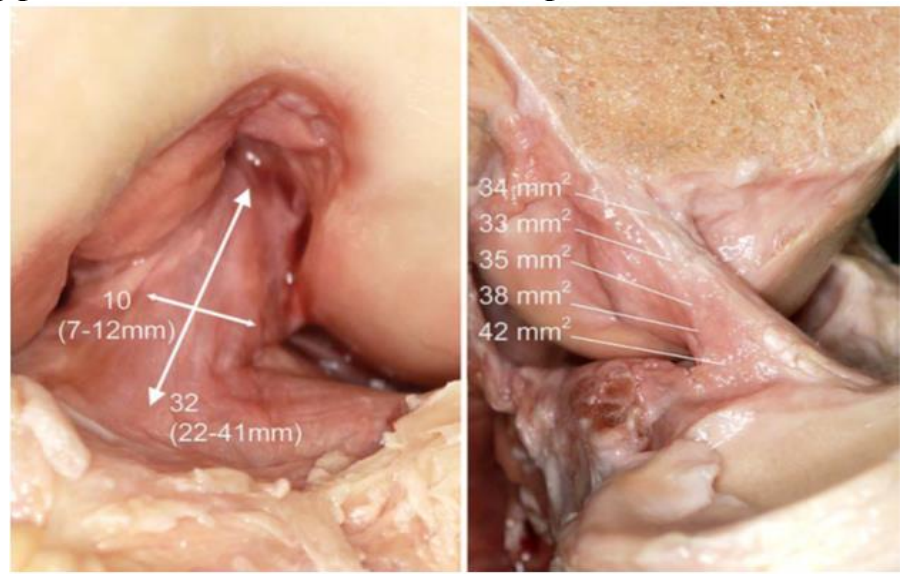

Fig.(4): The mean length is $32 \mathrm{~mm}$ (range $22-41 \mathrm{~mm}$ ) (left picture) and the mean width is $10 \mathrm{~mm}$ (range, 7-12 mm). The cross sectional area varies in size and shape from the femur to the tibia (right picture) ${ }^{(18)}$.

\section{Biomechanics}

\section{BIOMECHANICS OF ACL}

Normal function of the knee relies on a complex interplay between motion and stability. An understanding of the biomechanics of the ACL can only occur in conjunction with that of the entire knee joint ${ }^{(19)}$.

The primary function of the ACL is to prevent anterior translation of the tibia relative to the femur. Other functions of the ACL include resisting internal rotation of the tibia and varus or valgus stress of the tibia in the presence of collateral ligament injury. Clinically, this function can be tested with the "pivot shift test" which involves applying a combined internal tibial and valgus torque throughout the range of flexion-extension ${ }^{(\mathbf{1 9})}$.

Fibers from the posterolateral and anteromedial bundles are thought to be recruited differentially as the knee moves through a full range of motion. The posterolateral bundle of the ACL is 
thought to function as the principle restraint to anterior translation from 0 degrees to approximately 45 degrees of flexion, while the anteromedial bundle is thought to function as the principle restraint at higher flexion angles (Fig.5) ${ }^{(20)}$.

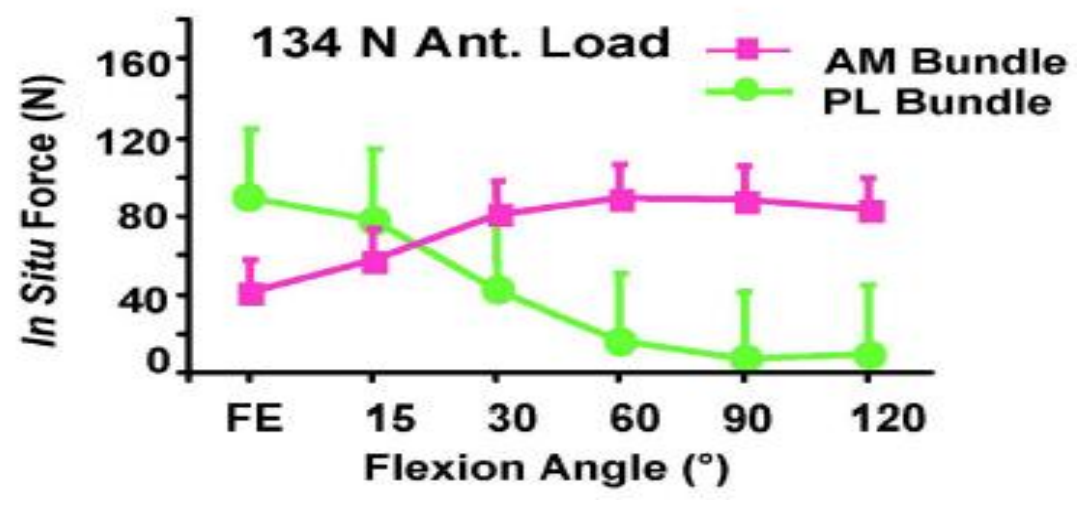

Fig. (5): Magnitude of the in situ force in the intact AM bundle and PL bundle in response to $134 \mathrm{~N}$ anterior tibial $\operatorname{load}^{(21)}$.

Based on in vitro studies, the anteromedial bundle has been observed to shorten from 0 degrees to 30 degrees of flexion, return to its baseline length from 30 degrees to 70 degrees of flexion, and lengthen from 70 degrees to 120 degrees of flexion. Conversely, the posterolateral bundle has been observed to reach maximum length with the knee in full extension and experience progressive shortening as the knee flexes, which together with the observed lengthening of the anteromedial bundle at high flexion angles supports the notions of differential recruitment and reciprocal function ${ }^{(20)}$.

In vivo studies have shown muscular activation to significantly increase ACL strain. In particular, quadriceps activity produces a marked increase in ACL strain, most pronounced in full extension. Gastrocnemius activity has also been shown to increase ACL strain at low flexion angles, yielding a cumulative strain during co-contraction with the quadriceps that is significantly greater than that produced by the quadriceps alone. Hamstring contraction decreases ACL strain at all flexion angles, but may not provide significant protection at very low flexion angles given the decrease in mechanical advantage experienced near full extension ${ }^{(22)}$.

Beynnon and colleagues ${ }^{(19)}$ have generated the most comprehensive characterization of in vivo strain experienced by the ACL. Using a Hall effect transducer implanted in the anteromedial bundle of the ACL, Beynnon and colleagues directly measured strains during a wide variety of maneuvers (Fig. 5).

The highest recorded strains were during isometric quadriceps contraction $\left(30 \mathrm{~N} / \mathrm{m}^{2}\right.$ extension torque) at 15 degree flexion ${ }^{(\mathbf{1 9}, 23)}$.

Anterior shear loads applied at $30^{\circ}$ of flexion (Lachman test) produced significantly greater strain than similar loads applied at 90 degrees of flexion (anterior drawer test), which correlates with the acknowledged superior clinical sensitivity of the Lachman test over the anterior drawer test in the detection of ACL injury. Limiting the clinical significance of these findings, however, is that strain measurements were isolated to the anteromedial bundle of the ACL ${ }^{(24)}$.

Noyes et al. ${ }^{(25)}$ demonstrated that native ACL had an ultimate load to failure of approximately 1,725 $\mathrm{N}$.

\section{Structural properties of the bone-ligament-bone complex:}

The structural behavior of the bone-ligamentbone complex can be described by a load-deformation curve. The curve consists of different characteristic regions. Initially, little load is required to elongate the ligament. This is characterized by the relatively flat "toe" region of the curve. Stiffness, defined as the slope of the load-deformation curve, is low. This region of the curve can be attributed to the "crimp" and "recruitment" pattern of the ligament ${ }^{(26)}$.

During tensile stretch, fibril "crimp" is first straightened out by small loads, after which larger loads are needed to elongate these fibrils. As such, an increasing number of fibrils become load bearing as larger loads are applied "recruitment"' and a gradual increase in tissue stiffness is seen, resulting in a nonlinear load-elongation curve ${ }^{(26)}$.

The change from the toe to the linear portion of the curve represents the change in stiffness that an examiner perceives during a clinical laxity examination when a ligament's endpoint is reached. The toe region is followed by a second, high stiffness region where significantly larger loads are required for continued elongation. Here, all the collagen fibers are straightened, and the curve becomes linear. This region characterizes the elastic deformation of the ligament ${ }^{(27)}$. 
If loading continues past the yield point, there is a sudden loss in the ability of the ligament to transmit load. Plastic deformation occurs until ultimate (failure) load is reached, when a sudden drop in load is recorded, representing total failure of the ligament. The area under the load-deformation curve represents the amount of energy absorbed by the ligament during testing ${ }^{(27)}$.

\section{Complications}

\section{Intraoperative complications:}

Complications include anesthetic complication, vascular and nerve injury, compartment syndrome and cartilage injury during arthroscopy and graft failure (28).

\section{Anasthesia complication}

The most frequent anesthetic complications include nausea and vomiting with general anesthetics and spinal headaches with regional anesthetics (28). Adult respiratory distress syndrome requiring hospitalization has been reported. Careful preoperative evaluation of patients limits perioperative anesthetic complications ${ }^{(29)}$.

\section{Neuro vascular injury}

When establishing new portals, use transillumination to demonstrate vascular structures. Vascular injuries associated with arthroscopic anterior cruciate ligament reconstruction (ACLR) are uncommon injuries. vascular complications following arthroscopic ACLR, formation of pseudo aneurysms, thrombosis or emboli of popliteal artery are presented. The mechanism of vascular injury is proposed to be associated with drilling of either tibial or femoral tunnels and direct damage of bony spikes. The vascular and nerve injury are very rare except for the infrapatellar nerve branch of the saphenous nerve which is inevitably damaged during midline prepatellar incision during harvesting BPTB graft and Graft failure ${ }^{(30)}$.

\section{ACL Graft Failure}

Patients who have had an ACL reconstruction can accidentally re-injure their knee and tear the graft (fig. 6), failure is more often due to factors over which the surgeon has control.

The tragedy is that the primary procedure offers the best chance to obtain a fully functional and stable knee. Once the patient has had surgery, and then struggled to rehabilitate in the face of non-optimal stability, then struggled again after failure of the repair, the whole situation becomes a great deal more complicated, The menisci take more stress and can become secondarily damaged, The joint surface becomes damaged and arthritis can become established, The muscles become weak and the bone stock becomes poor ${ }^{(31)}$.

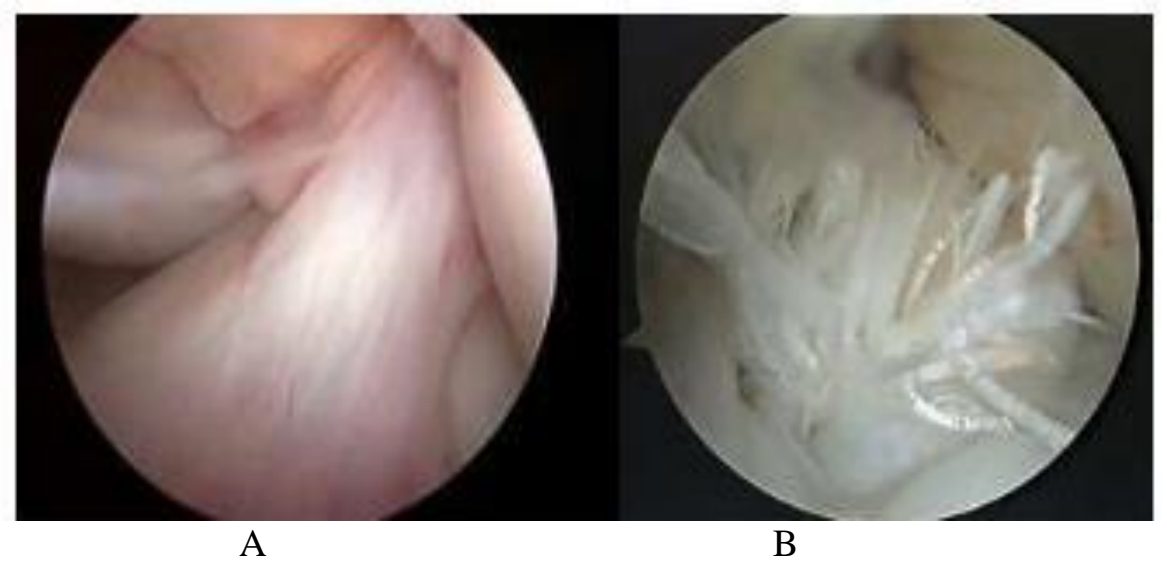

Fig. (6): (A) scope image of normal ACL (B) Graft failure ${ }^{(31)}$.

\section{1 - ACL graft failure due to inadequate graft material $(\mathbf{5 \%})$}

The strength of the graft and care in graft preparation prior to implantation are important to the success of the primary ACL procedure, and so is the complex revascularisation and remodelling of the graft once it is implanted ${ }^{(32)}$.
In considering the reasons why an ACL graft might fail, there are a number of points about the graft material itself:

a-Inappropriate use of primary repair

b-Poor structural mechanical property of the graft prior to implantation

c-Allografts

d-Synthetic grafts

e-Autograftsue: 


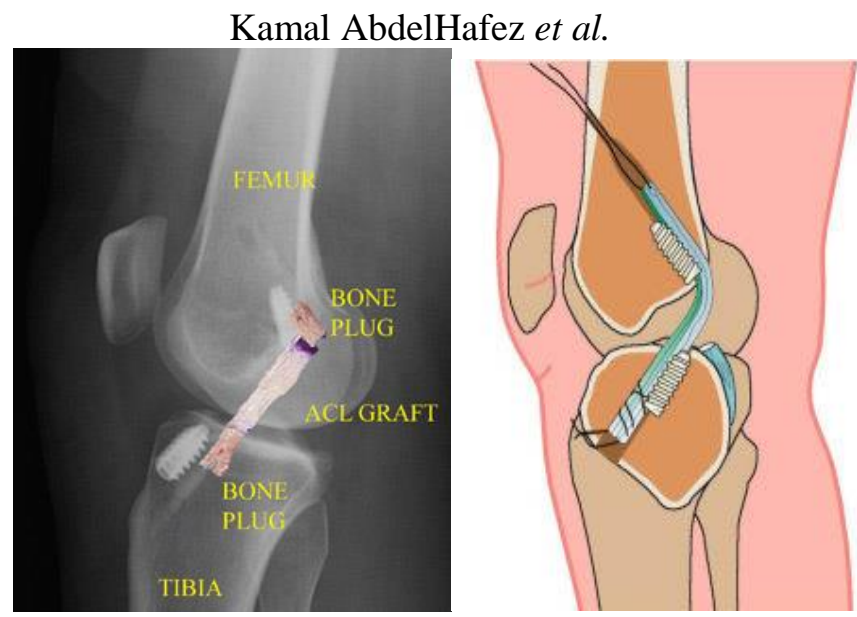

A

B

Fig. (7): (A) Post-operative X-ray after ACL patellar tendon reconstruction (with picture of graft superimposed) shows graft position and bone plugs fixation with metal interference screws. (B) Post-operative picture after ACL

f- Failure of ACL graft ligamentisation reconstruction shows graft position and fixation with metal interference screws ${ }^{(33) .}$

2 - ACL graft failure due to graft impingement

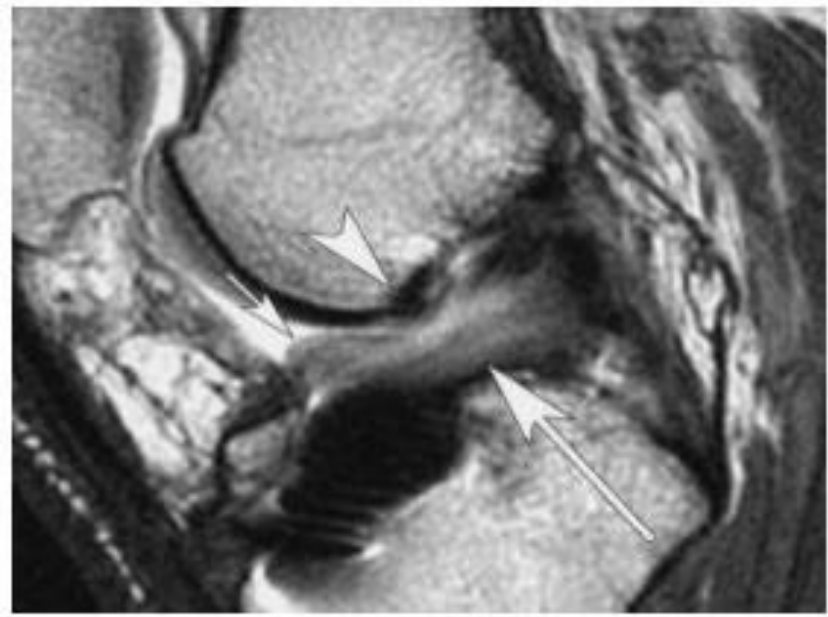

Fig. (8): MR arthrogram shows increased signal intensity in graft (long arrow). Spur (arrowhead) at anterior margin of intercondylar notch deforms the superior surface of the graft, which bulges (short arrow) anterior to the spur ${ }^{(34)}$. Graft impingement mainly occurs when the graft becomes trapped in the intercondylar notch of the femur (fig. 8).

This can cause graft abrasion on the walls and edges of the notch, with wear and tear and eventual graft failure. The frayed edges can become bunched up and obstruct movement of the joint, causing decreased range of motion. May cause pain or loss of extension (34)

\section{Causes of graft impingement}

Causes of ACL graft impingement include -

\section{Position of the femoral and tibial tunnels}

Faulty operative technique is frequently a significant factor in failure of an ACL graft procedure, with misplaced femoral or tibial tunnels topping the list of causes of graft failure. 


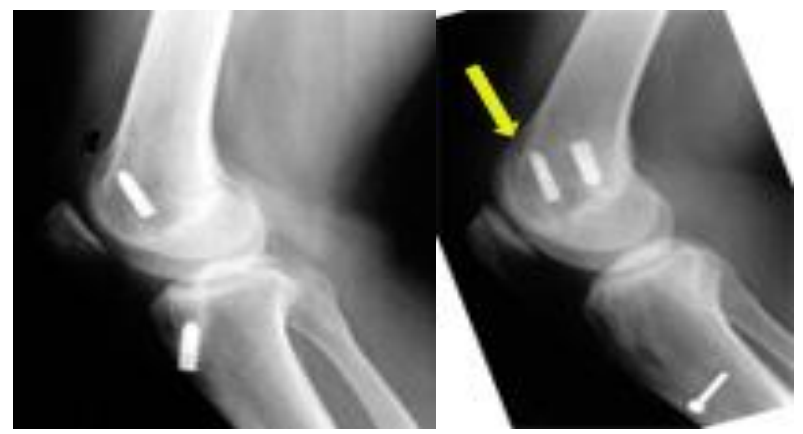

Fig. (9): This left X-ray shows the joint where the ACL graft failed because the tunnels were too anterior. Take a look at the femur (top bone). The white bits are the fixation devices holding the graft in the tunnel. The right $\mathrm{X}$-ray shows the original tunnel (yellow arrow) with the new tunnel behind it and in the correct position. You get an idea of how far anterior the original tunnel was made. If you cannot orientate, look for the patella bone -

\section{Treatment:} that is the anterior aspect ${ }^{(34)}$.

Acute compartment syndrome is an emergency requiring immediate surgical treatment, known as a fasciotomy (fig. 10), to allow the pressure to return to normal ${ }^{(35)}$.

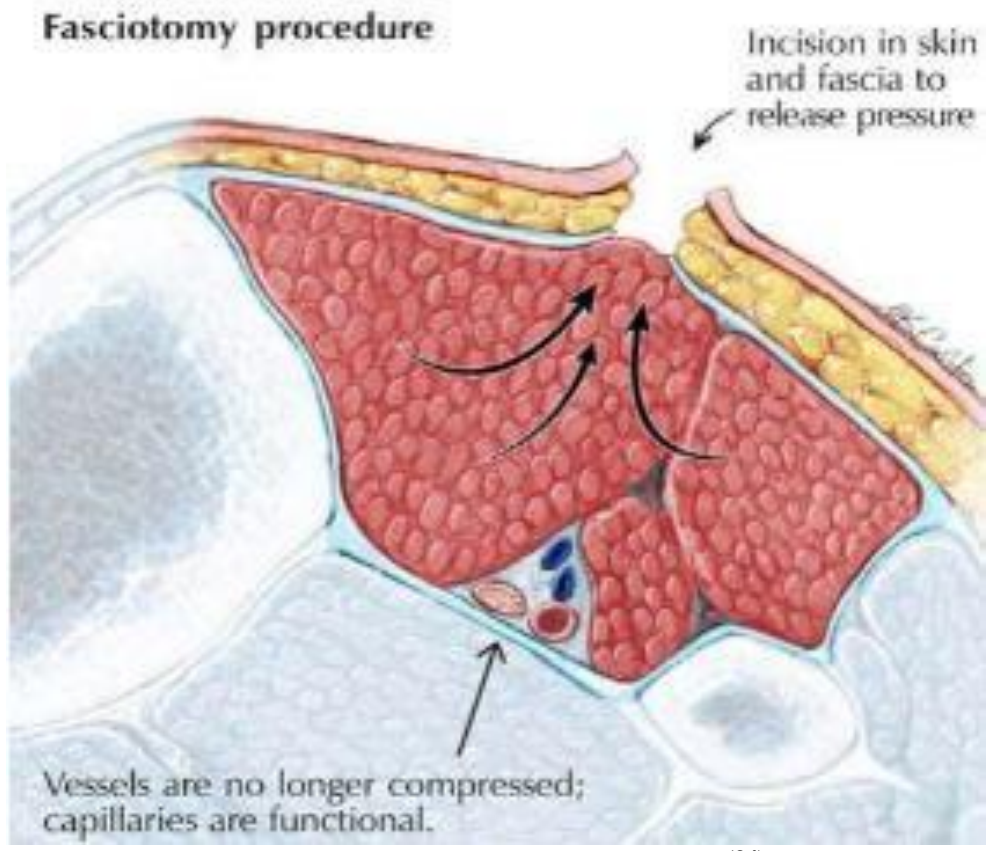

Fig. (10): Fasciotomy procedure ${ }^{(34)}$.

When compartment syndromes are suspected, the important immediate measure is wide-splitting of any constricting dressings that have been applied, icing the leg and elevation of the limb. Surgery is the treatment of choice, which requires long incisions of the skin and fascia, splitting of the retinaculae, excision of necrotic tissue and evacuation of the hematoma. The skin is left open and closed 2-8 days later. The fasciotomy could be done with a single incision, or double incisions ${ }^{(33)}$. The single incision is over the lateral aspect of fibula, while the double incisions include both lateral and postero-medial incisions. The incisions could be short $(4-8 \mathrm{cms})$, or long incisions $(15-30 \mathrm{cms})$ described a mechanical method of fasciotomy wound closure that is effective in 24 days, has described a single incision, four compartment fasciotomy of the leg. Skin grafts may be required to close the wound ${ }^{(32)}$.

Subacute compartment syndrome, while not quite as much of an emergency, usually requires urgent surgical treatment similar to acute compartment syndrome.Hyperbaric oxygen therapy has been shown to be a useful adjunctive therapy for compartment syndrome, and other acute traumatic ischemias by improving wound healing and reducing repetitive surgery ${ }^{(29)}$.

\section{Prevention}

There is probably no way to prevent this condition, however, early diagnosis and treatment will help prevent many of the complications. Persons with casts need to be made aware of the risk of swelling and 
Kamal AbdelHafez et al.

should see their health care provider or go to the emergency room if pain under the cast increases despite pain medicines and raising the area ${ }^{(34)}$.

\section{Complications}

Failure to relieve the pressure can result in necrosis of tissue in that compartment, since capillary perfusion will fall leading to increasing hypoxia of those tissues. This can cause Volkmann's contracture in affected limbs.

If left untreated, acute compartment syndrome can lead to more severe conditions including rhabdomyolysis, kidney failure and an infection may develop. A possible complication of surgical intervention for compartment syndrome can be chronic venous insufficiency. In more severe cases, amputation may be required.

\section{Conclusion:}

The challenge is to perform the reconstructive procedure with high success and minimal complications and morbidity. This requires a combination of a skilled surgeon, appropriate graft selection and careful attention to the details of rehabilitation.

\section{References}

1. Aichroth $P$ (1993): Osteochondritis Dissecans of the Knee. J. Bone Joint Surg. Br., 73:461-464.

2. Burman MS (1931): Arthroscopy or Direct Visualization of Joints: An Experimental Cadaver Study. J. Bone Joint Surg., 13:669712.

3. Applegate GR, Flannigan BD, Tolin BS et al. (1993): MR Diagnosis of Recurrent Tears in the Knee. The Value of Intraarticular Contrast Material. Am. J. Roentgenol., 161:821-832.

4. Bradley J and Dandy D (1989): Results of Drilling Osteochondritis Dissecans Before Skeletal Maturity. J. Bone Joint Surg., 71:642655.

5. Han Y, Sardar Z, McGrail S et al. (2011): Peri-anterior cruciate ligament reconstruction femur fracture: a biomechanical analysis of the femoral tunnel as a stress riser. Knee Surg. Sports Traumatol. Arthrosc., 19:77-85.

6. Milankov MZ, Miljković $\mathbf{N}$ and Janjić $\mathbf{N}$ (2010): Hybrid hamstring graft tibial fixation in anterior cruciate ligament reconstruction. Arch. Orthop. Trauma Surg., 130:1033-1036.

7. Girgis F, Marshall J and Jem A (2017): The Cruciate Ligaments of the Knee Joint: Anatomical. Functional and Experimental Analysis. Clin. Orthop. Relat. Res., 106:216231.
8. Petersen W and Zantop T (2006): Anatomy of the Anterior Cruciate Ligament with Regard to Its Two Bundles. Clinical Orthopaedics and Related Research, 454: 3547.

9. Petersen W, Unterhauser F, Pufe T et al. (2003): The angiogenic peptide vascular endothelial growth factor (VEGF) is expressed during the remodeling of free tendon grafts in sheep. Arch. Orthop. Trauma. Surg., (123): 168-174.

10. Starman J, Ferretti M, Järvelä T et al. (2007): Anatomy and Biomechanics of the Anterior Cruciate Ligament. Orthop. Clin. North Am., 33(4):605-620.

11. Siegel L, Albanese $C$ and Siegel D (2012): Anterior Cruciate Ligament Injuries: Anatomy, Physiology, Biomechanics, and Management. Clin. J. Sport Med., (22):349355.

12. Giuliani J, Kilcoyne $K$ and Rue J (2009): Anterior cruciate ligament anatomy: A review of the anteromedial and posterolateral bundles. J. Knee Surg., 22:148-154.

13. Beasley L, Weiland D, Vidal A et al. (2005): Anterior Cruciate Ligament Reconstruction: A Literature Review of the Anatomy, Biomechanics, Surgical Considerations, and Clinical Outcomes. Oper. Tech. Orthop., 15:519.

14. Harner C, Livesay G, Kashiwaguchi S et al. (1995): Comparative study of size and shape of human anterior and posterior cruciate ligaments. J. Orthop. Res., 13: 429-434.

15. Dienst $M$, Burks $R$, Greis $P$ et al. (2002): Anatomy and biomechanics of the anterior cruciate ligament. Orthop. Clin. N. Am., 33: 605-620.

16. Edwards T, Guanche $\mathrm{C}$, Petrie $\mathrm{S}$ et al. (1999): In vitro comparison of elongation of the anterior cruciate ligament and single and dual tunnel anterior cruciate ligament reconstructions. Orthopedics, 22: 577-584.

17. Woo $\mathrm{S}$, Wu $\mathrm{C}$, Dede $\mathrm{O}$ et al. (2006): Biomechanics and anterior cruciate ligament reconstruction. Journal of Orthopaedic Surgery and Research, 2: 1-9.

18. McCarty $L$ and Bach B (2005): Anatomy, Biology and biomechanics of patellar tendon autograft anterior cruciate ligament reconstruction. Techniques in Orthopaedics, 20(4):342-352.

19. Beynnon B, Johnson R, Fleming $B$ et al. (1997): The strain behavior of the anterior cruciate ligament during squatting and active flexion-extension. A comparison of an open 
Anatomy, Biomechanics and Complications of Arthroscopic Anterior Cruciate Ligament Reconstruction

and a closed kinetic chain exercise. Am. J. Sports Med., 25:823- 829.

20. Fleming B, Renstrom P, Beynnon B et al. (2001): The effect of weight bearing and external loading on anterior cruciate ligament strain. J. Biomech., 34:1663-1670.

21. Fleming B, Renstrom $\mathbf{P}$ and Ohlen $\mathbf{G}$ (2001): The gastrocnemius muscle is an antagonist of the anterior cruciate ligament. J. Orthop. Res., 19: $1178-1184$.

22. Gan TJ, Meyer TA, Apfel CC (2007): Society for ambulatory anesthesia guidelines for the management of postoperative nausea and vomiting. Anesth. Analg., 105: 16151628.

23. Williams BA, Dang Q, Bost JE (2009): General health and knee function outcomes from seven days to twelve weeks after spinal anesthesia and multimodal analgesia for anterior cruciate ligament reconstruction. Anesth. Analg., 108: 1296-1302.

24. Uzümcügil O, Doğan A, Yalçinkaya M et al. (2009): Clinical importance of femoraland tibial tunnel localizations in arthroscopic anterior cruciate ligament reconstruction. Eklem. Hastalik. Cerrahisi., 20:25-31.

25. Noyes FR and Barber-Westin SD (2001): Revision anterior cruciate surgery with use of bone-patellar tendon-bone autogenous grafts. J. Bone Joint Surg. Am., 83:1131-1143.

26. Wetzler M, Bartolozzi A, Gillespie M et al. (1996): Revision anterior cruciate ligament reconstruction. Oper. Techn. Orthop., 6:181192.

27. Gretelman MH and Friedman MJ (1999): Revision anterior cruciate ligament reconstruction surgery. Journal of the
American Academy of Orthopaedic Surgeons, 7(3): 189-198.

28. Zantop T, Diermann N, Schumacher T et al. (2008): Anatomical and nonanatomical double-bundle anterior cruciate ligament reconstruction: importance of femoral tunnel location on knee kinematics. Am. J. Sports Med., 36(4):678-685.

29. Harner CD, Giffin JR, Dunteman RC et al. (2000): Evaluation and treatment of recurrent instability after anterior cruciate ligament reconstruction. J. Bone Joint Surg. Am., 82:1652-1659.

30. Cooper GG (2002): A method of single incision, Four compartment fasciotomy of the leg. Eur. J. Vasc. Surg., 6(6):659-661.

31. Salcido $R$ and Lepre SJ (2007): Compartment syndrome: wound care considerations. Adv. Skin Wound Care, 20(10): 559-65.

32. McKenney MG, Nir I, Fee T et al. (2006): A simple device for closure of fasciotomy wound. Am. J. Surg., 172(3):275-277.

33. Bouachour G, Cronier P, Gouello JP et al. (2006): Hyperbaric oxygen therapy in the management of crush injuries: A randomized double-blind placebo-controlled clinical trial. J. Trauma, 41(2): 333-339.

34. Geiderman JM (2006): General principles of orthopedic injuries. In: Marx J, ed. Rosen's Emergency Medicine: Concepts and Clinical Practice. 6th ed. St Philadelphia, Pa: Mosby Elsevier: Chap 46. https://www.elsevier.com/books/rosensemergency-medicine-concepts-and-clinicalpractice-2-volume-set/marx/978-1-45570605-1 